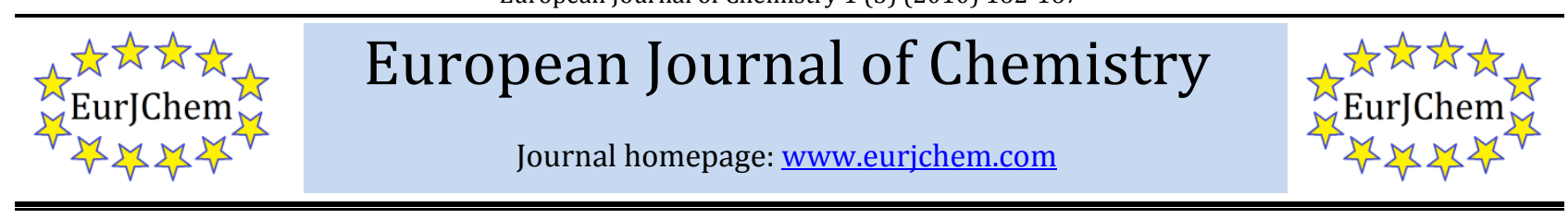

\title{
On the structure of liquid methyl salicylate: The role of intramolecular hydrogen bonding
}

\author{
Santiago Aparicio* and Rafael Alcalde \\ Department of Chemistry, University of Burgos, 09001 Burgos, Spain \\ *Corresponding author at: Department of Chemistry, University of Burgos, 09001 Burgos, Spain. Tel.: $+34947258062 ;$ fax: +34947258831.

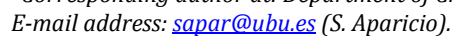

\section{ARTICLE INFORMATION}

Received: 03 March 2010

Received in revised form: 11 May 2010

Accepted: 22 June 2010

Online: 30 September 2010

\section{KEYWORDS}

\section{Salicylate}

Hydrogen bonding

DFT-Molecular dynamics

Vibrational spectroscopy

Relaxation spectroscopy

\section{ABSTRACT}

A study on the structure of methyl salicylate is reported using quantum mechanical calculations, molecular dynamics simulations, vibrational spectroscopy and microwave dielectric relaxation spectroscopy tools. The reported results show that a strong intramolecular hydrogen bonding is developed between the hydroxyl hydrogen and carbonyl oxygen. This intramolecular interaction is maintained in gas and liquid phases and even when diluted in inert solvents. Interaction between neighbour molecules is developed through dipolar interactions, and thus, intermolecular hydrogen bonding should be discarded for pure liquid methyl salicylate. The interaction between neighbour methyl salicylate molecules do not lead to remarkable changes in the intramolecular hydrogen bonding.

\section{Introduction}

In a previous work [1], we reported a wide study on the properties of aromatic esters in the liquid state using a collection of experimental and computational tools to infer the molecular level features that characterized their liquid phase structure and properties. As a continuation of that study, we report here a wider analysis of liquid phase properties of Methyl Salicylate (MS, CAS number 9041-28-5, Scheme 1). MS is a molecule characterized by the close proximity of hydroxyl and ester groups, leading to a strong intramolecular hydrogen bonding. The question that arises is the effect of possible competing intermolecular hydrogen bonding between neighbour molecules with the strong intramolecular interaction, that is to say, does the development of intermolecular interactions weaken the intramolecular hydrogen bonding? The likely development of intermolecular hydrogen bonding between neighbour MS molecules should lead to an out-of-plane movement of the hydroxyl group leading to a weakening of intramolecular interaction. This weakening of intramolecular hydrogen bonding for the effective development of intermolecular interactions has been reported by our group for other families of ester molecules such as ethyl lactate [2]. Several literature studies have proposed that intramolecular hydrogen bonding remains almost unaffected in the liquid phase for pure MS, and thus, no competing effect of possible intermolecular interactions may be inferred. Yamada [3] studied the properties of $\mathrm{MS}$ in $\mathrm{CCl}_{4}$ through infrared spectroscopy showing strong intramolecular hydrogen bonding between hydroxyl hydrogen and carbonyl oxygen. Wojcik and Paluszkiewicz [4] analyzed the infrared spectra of MS and showed that MS molecules form intramolecular hydrogen bonding in gas, liquid and solid phases. Berthelot et al. [5] showed that mixing MS with octanol or dimethylsulfoxide does not lead to remarkable changes for intramolecular hydrogen bonding in MS molecules. Mitsuzuka et al. [6] analyzed the OH stretching vibration of MS, showing a weak and broad band because of the intramolecular hydrogen bonding, this band showed similar characteristics for small size clusters formed with molecules such as water or methanol, discarding the transformation of intra to intermolecular hydrogen bonding. Palomar et al. [7] reported a vibrational study of intramolecular hydrogen bonding in MS which was discussed together with quantum mechanical calculations, the results showed a fair agreement between experimental and calculated results with bands assigned considering intramolecular hydrogen bonding between the hydroxyl and carbonyl groups. Melandri et al. [8] reported a rotational spectroscopy study on MS showing that the ground electronic state of this molecule adopts a form stabilized through the development of intramoleculecular hydrogen bonding between the hydroxyl and carbonyl groups. Massaro et al. [9] reported a vibrational analysis of MS isomers showing that the lowest energy isomer is the MS1 form (Scheme 1), in which intramolecular hydrogen bonding between hydroxyl hydrogen and carbonyl oxygen is developed. These authors also showed that the form MS2 (Scheme 1), in which hydrogen bonding is developed between the hydroxyl hydrogen and the methoxy oxygen moieties is also very stable. We reported in a previous study [1] results in agreement with those reported by the remaining authors showing that the MS1 form, Scheme 1, is the most stable isomer and that the transformations between the different isomers evolves through large torsional barrier because of the strength of the intramolecular hydrogen bonding. We reported that the MS2 isomer is only $2.95 \mathrm{kcal}$ mol-1 $^{-1}$ less stable than MS1 form, but the transformation between both isomers evolves through a very large torsional barrier (13.54 $\left.\mathrm{kcal} \mathrm{mol}^{-1}\right)$, therefore we may expect that if MS2 isomer appears it will remain in this form because of the large energy required to evolve through the more stable MS1 form. 
<smiles>CCOC(=O)c1ccccc1</smiles>

MS1<smiles>CCOC(=O)c1ccccc1</smiles>

MS2

In this work we will analyze the structure of liquid MS using four different experimental / computational approaches: i. Quantum mechanical calculations of gas phase isolated molecules, ii. Molecular dynamics simulations in liquid phase, iii. Liquid phase vibrational spectroscopy and $i v$. Liquid phase microwave dielectric relaxation spectroscopy. The main objective of the work is to analyze the competing role of intermolecular hydrogen bonding between neighbour MS molecules and its effect on the strength and characteristics of intramolecular hydrogen bonding.

\section{Experimental and Computational Methods}

These methods have been described in deep in previous works, and thus they will be only briefly explained in this work. Quantum mechanical calculations were performed on isolated MS monomers in gas phase with the Gaussian 03 package [10] according to Density Functional Theory (DFT) approach, with the Becke gradient corrected exchange functional [11] and LeeYang-Parr correlation functional [12] with three parameters (B3LYP) [13] method, 6-311++G** basis set was used along this work. Atoms in a molecule (AIM) calculation [14] were carried out using the AIM2000 program [15]. Classical molecular dynamics simulations (MD) were carried out using the TINKER molecular modelling package [16], with all simulations performed in the NPT ensemble. MS molecules were described according to the OPLS-AA force field [17] for molecular dynamics simulations, force field parameterization and details of the simulation may be found in a previous work [1].

Attenuated total reflection infrared (ATR-FTIR) spectroscopy studies were developed with a Nicolet Nexus spectrometer together with a Smart Thermal ARK device. The ATR accessory contains a Zinc Selenide crystal, which temperature is controlled through a built-in controller, and measured through a RTD temperature sensor to $\pm 1{ }^{\circ} \mathrm{C}$. Microwave dielectric relaxation spectroscopy (DRS) measurements were performed according to a coaxial reflection technique using a vector network analyzer (Agilent $\mathrm{N} 5230 \mathrm{~A}$ ) in the $200 \mathrm{MHz}-20 \mathrm{GHz}$ frequency range with an Agilent 85070E dielectric probe kit and an Agilent N4691B ECal electronic calibration module; the cell temperature was controlled with an external circulating bath and measured to

$\pm 1 \cdot 10^{-2} \mathrm{~K}$ with a platinum resistance thermometer, the measurement and calibration procedures were previously reported [18].

\section{Results and Discussion}

AIM analysis of B3LYP $/ 6-311++G^{* *}$ results obtained for MS monomer in gas phase is reported in Table 1 and Figure 1. According to the AIM approach, two main criteria must be fulfilled to define a true hydrogen bonding $[19,20]: i)$ a bond path between two atoms, with the existence of a bond critical point, $\mathrm{BCP}$, in the middle of the path and ii) the electron density at BCP, $\rho_{\mathrm{BCP}}$, and the laplacian of that electron density, $\nabla^{2} \rho_{B C P}$, must be within the 0.002-0.035 and 0.024-0.139 ranges, respectively, (both in atomic units). A bond path is reported in Figure 1 for the interaction between hydroxyl hydrogen and carbonyl oxygen with a BCP (BCP-H_bonding) close to the hydrogen donor. Moreover, a ring critical point (RCP) is also obtained, thus, reinforcing the idea of a strong intramolecular hydrogen bond for the most stable MS isomer, Figure 1 . The values of $\rho_{\mathrm{BCP}}$, and $\nabla^{2} \rho_{B C P}$ reported in Table 1 are in the high limit of the requirements to define a hydrogen bond, and thus, a strong interaction may be inferred.

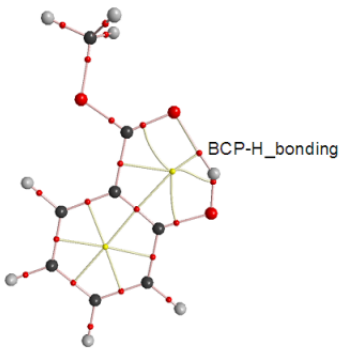

(a)

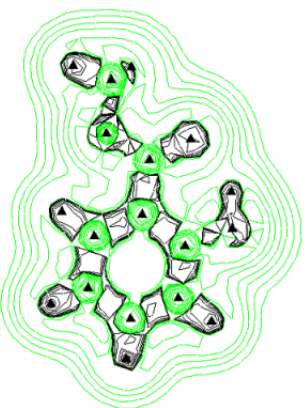

(b)
Figure 1. AIM analysis for MS. (a) Molecular graph; small red dots represent bond critical points (BCP), small yellow dots represent ring critical points (RCP), pink lines represent bond paths, yellow lines represent ring paths, large dots represent attractors (atoms; black: carbon, gray: hydrogen, red: oxygen). (b) Contour plot for the Laplacian of electron density, $\nabla^{2} \rho$; green curves represent positive isosurface values of $\nabla^{2} \rho$ and black curves represent negative isosurface values of $\nabla^{2} \rho$. In panel a, we show BCP corresponding to intramolecular hydrogen bonding (BCP-H_bonding)

The energetic properties of BCP associated with the intramolecular hydrogen bonding also provide valuable information about the characteristics of this interaction. Kinetic energy density, $G$, is always positive, and its ratio to electron density, $G / \rho_{B C P}$, may be used to define the character of the interaction, considering that it use to be larger than 1.0 for closed shell (hydrogen bonding, ionic bonds and van der Waals interactions) and less than 1.0 for shared interactions (covalent bonds) [14]. Potential energy density (or virial field), $V$, that is always negative, is related to the covalency of the interaction [21] and with hydrogen bonding strength through several empirical relationships [22,23]. Moreover, total energy density, $H$, the sum of $G$ and $V$, should be positive for closed shell interactions, such as hydrogen bonding, indicating that kinetics energy density dominates the potential energy density, and negative for shared interactions [14]. The ratio $G / \rho_{B C P}$ reported in Table 1 is lower than 1.0 for the intramolecular hydrogen bonding, although for closed shell interactions as these ones they should be larger than 1.0; nevertheless, values slightly lower than 1.0 have been previously reported for remarkably strong hydrogen bondings in other compounds 
Table 1. AIM analysis of MS computed in gas phase at the B3LYP/6-311++ $\mathrm{G}^{*}$ theoretical level. Electron density at BCP (BCP-H_bonding, Figure 1), $\rho_{\mathrm{BCP}}$, laplacian of electron density at BCP, $\nabla^{2} \rho$ BCP, donor-acceptor interatomic distance, $r$, eigenvalues, $\lambda_{i}$, of hessian of electron density, ellipticity, $\varepsilon$, kinetic energy density, $G$, potential energy density (or virial field), $V$, total energy density, $H$, and ratio between $G$ and electron density at BCP, computed at the BCP corresponding to intramolecular hydrogen bonding. Values calculated for optimized structure reported in Figure 1.

\begin{tabular}{ccccccccccc}
\hline$\rho_{\text {BсP }} /$ a.u. & $\nabla^{2} \rho /$ a.u. & $\boldsymbol{r} / \AA$ & $\boldsymbol{\lambda}_{\mathbf{1}}$ & $\boldsymbol{\lambda}_{\mathbf{2}}$ & $\boldsymbol{\lambda}_{\mathbf{3}}$ & $\boldsymbol{\varepsilon}$ & $\boldsymbol{G}$ & $\boldsymbol{H}$ & $\boldsymbol{V}$ & $\boldsymbol{G} / \boldsymbol{\rho}_{\text {BCP }}$ \\
\hline 0.040 & 0.136 & 1.78 & -0.063 & -0.062 & 0.263 & 0.016 & 0.036 & -0.001 & -0.037 & 0.90 \\
\hline
\end{tabular}

[21]. $H$ value is almost null, pointing again to strong hydrogen bond. As a rule, the larger the $G$ values, the stronger the interaction, and thus, a strong intramolecular hydrogen bond may be inferred from the value reported in Table 1. Moreover, the $\nabla^{2} \rho_{B C P}$ plot reported in Figure $1 \mathrm{~b}$ shows a clear charge concentration between hydrogen bond donor and acceptor atoms for the intramolecular hydrogen bond. We should remember that $\nabla^{2} \rho_{B C P}<0$ shows charge concentration, whereas $\nabla^{2} \rho_{B C P}>0$ indicates charge depletion. The large charge depletion between hydroxyl hydrogen and carbonyl oxygen corresponds to the strong hydrogen bond, however the charge concentration on the hydrogen is not strongly polarised toward oxygen.

Although DFT / AIM analysis performed in the previous paragraphs supports remarkable conclusions on the characteristics of intramolecular hydrogen bonding, these calculations were performed on isolated gas phase MS monomers, and thus the effect of surrounding molecules should be considered. To study these effects classical molecular simulations were performed to analyze the behavior of liquid phases; relevant results are reported in Figure 2. The first structural feature inferred from MD simulations is the relative position of neighbour hydroxyl and carbonyl groups. Results reported in Figure 2 shows the time evolution along simulations of two dihedrals, $\varphi_{1}$ and $\varphi_{2}$. The first dihedral, $\varphi_{1}$, shows if the atom close to hydroxyl oxygen is carbonyl oxygen or methoxy oxygen, that is to say, if intramolecular hydrogen bonding is developed through MS1 $\left(\varphi_{1}\right.$ close to $\left.0^{\circ}\right)$ or through the MS2 isomers $\left(\varphi_{1}\right.$ close to $\left.180^{\circ}\right)$. The results reported in Figure 2 shows that $\varphi_{1} \sim 10^{\circ}$ (in agreement with results reported by Melandri et al. [8]), thus pointing to a clear prevalence of MS1 isomer. Nevertheless, a small population of MS2 isomers $(<5 \%)$ is present along the simulations, this population of MS2 isomers remains almost constant maybe because of the large torsional barrier required for the transformation of MS2 to MS1 [1]. The second dihedral, $\varphi_{2}$, shows the position of $\mathrm{OH}$ group related to the aromatic ring and $\mathrm{COO}$ group, and thus, from this dihedral we may infer the effect of surrounding MS molecules on intramolecular hydrogen bonding. $\varphi_{2}$ values reported in Figure 2 are around $34^{\circ}$, and thus, neighbour molecules lead to a slight out-of-plane movement of hydroxyl hydrogen. Nevertheless, the value of $\varphi_{2}$ in liquid MS is small and thus the strong intramolecular hydrogen bonding is also present in liquid phases of MS. We have also computed the number of intermolecular hydrogen bonds per MS molecule in liquid MS. We define each occurrence within $2.85 \AA$ A no remarkable effect of considered angles, within reasonable values, was found on the extension of hydrogen bonding, and thus, a $60^{\circ}$ value was used for all the possible interactions. The calculated number of intermolecular hydrogen bonds per MS molecules is lower than 0.1, thus discarding remarkable intermolecular interactions between neighbour MS molecules. This result is in agreement with low value of intermolecular interaction energy previously reported for MS [1] which is only a $15 \%$ larger than the value for its parent molecule methyl benzoate for which intermolecular hydrogen bonding is not possible. We should remark that the development of intermolecular hydrogen bonding may be hindered by two main factors: $i$. The strength of intramolecular hydrogen bond, that hinders the out-of-plane movement of hydroxyl hydrogen required to develop effective intermolecular hydrogen bonding and ii. The almost planar shape of MS molecules that hinders the approach of neighbour MS molecules (steric hindrance). Therefore MD results shows that intramolecular hydrogen bonding is very slightly weakened in liquid phases but remarkable intermolecular hydrogen bonding should be discarded, and thus, interactions between neighbour molecules should be developed mainly through dipolar interactions as for its parent molecule methyl benzoate [1].

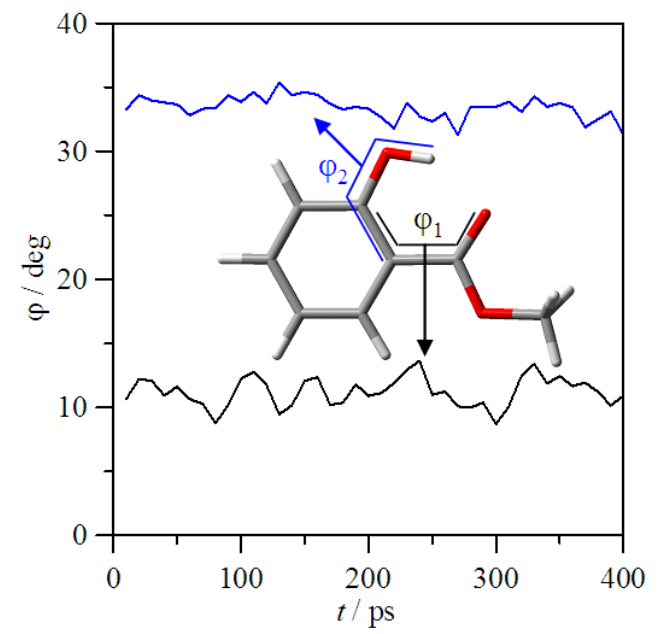

Figure 2. Time evolution of the reported dihedral angles obtained from molecular dynamics simulations at $298 \mathrm{~K} / 0.1 \mathrm{MPa}$.

A detailed theoretical analysis of IR results for MS was previously reported by Massaro et al. [9] and experimental data were reported by Varghese et al. [24]. ATR-FTIR results obtained in this work are reported in Figures 3-6.

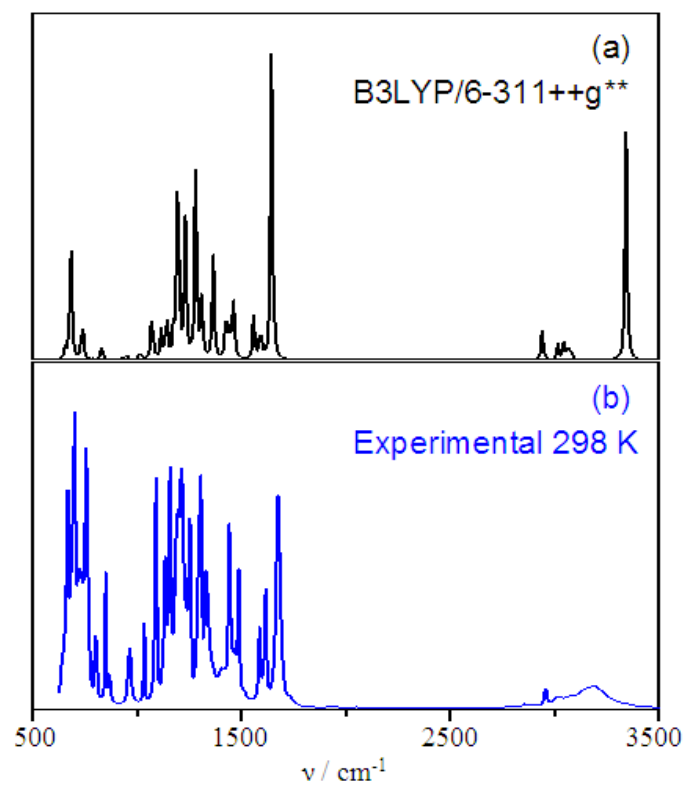

Figure 3. (a) Calculated and (b) experimental IR spectra of MS. Scaling factor for calculated spectrum is 0.96 . 

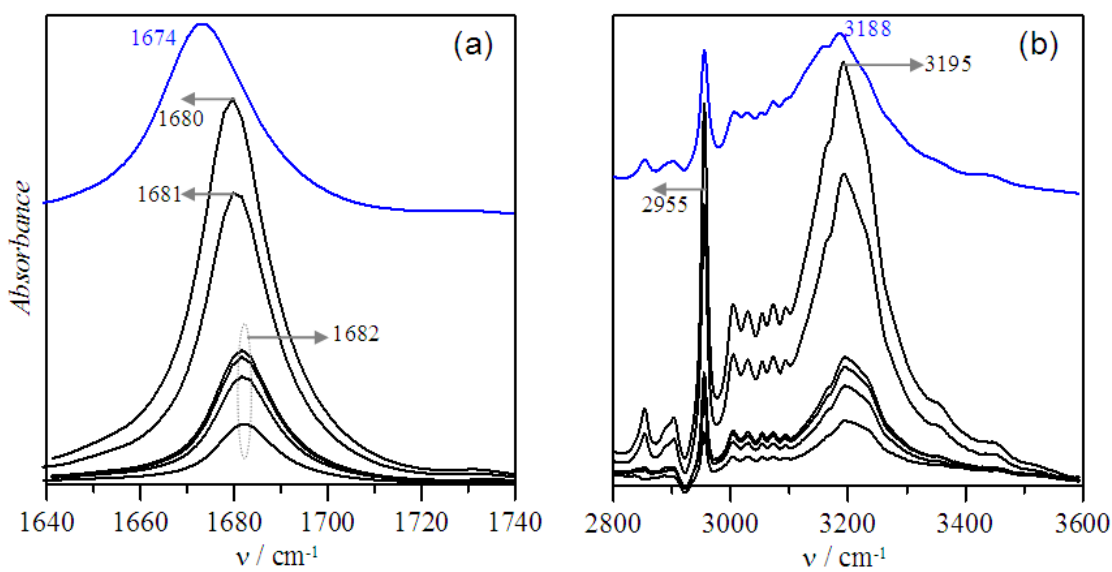

Figure 4. ATR-FTIR Spectra of $\mathrm{MS}$ in $\mathrm{CCl}_{4}$ at different concentrations, in the (a) $\mathrm{C}=\mathrm{O}$ and (b) $\mathrm{O}-\mathrm{H}$ regions. Blue line shows spectrum for pure MS. All experiments at $298 \mathrm{~K}$. For results in $\mathrm{CCl}_{4}$ (black lines): $x_{M S}=0.010,0.025,0.030$, $0.035,0.10$ and 0.15 (from bottom to top).

In Figure 3 we compare the experimental spectrum of pure MS in liquid phase compared with the gas phase calculated spectrum using B3LYP/6-311++G** approach, the experimental spectrum is well reproduced by the calculations, only in the high frequency region $\left(2800-3500 \mathrm{~cm}^{-1}\right)$ we may appreciate some differences. The gas phase calculated $\mathrm{O}-\mathrm{H}$ stretching vibration leads to a narrow, sharp and intense peak with maximum at $3341 \mathrm{~cm}^{-1}$, whereas for the experimental liquid spectrum a broad band with maximum at $3188 \mathrm{~cm}^{-1}$ is obtained. The peak at lower frequencies in both experimental liquid $\left(2955 \mathrm{~cm}^{-1}\right)$ and gas phase calculated $\left(2937 \mathrm{~cm}^{-1}\right)$ spectra are assigned to $\mathrm{CH}_{3}$ group. Another remarkable feature in the spectra of MS is the peak due to $\mathrm{C}=0$, which appears at 1642 $\mathrm{cm}^{-1}$ and $1674 \mathrm{~cm}^{-1}$, for gas and liquid phases, respectively. The $\mathrm{C}=\mathrm{O}$ signal is a narrow and sharp peak in contrast with the wide $\mathrm{C}=\mathrm{O}$ band for liquid $\mathrm{MS}$.

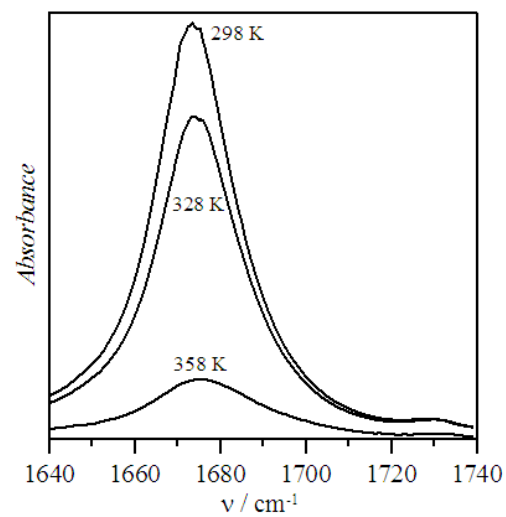

Figure 5. ATR-FTIR spectra of pure MS as a function of temperature.

To analyze the extension of intramolecular hydrogen bonding, ATR-FTIR spectra of MS solutions in $\mathrm{CCl}_{4}$ were recorder for different concentrations, Figure 4 . The analysis of the $\mathrm{C}=\mathrm{O}$ peak shows that the frequencies and shapes of this peak do not change remarkably with increasing MS concentration and they are almost equal to the peak in pure liquid MS. A slight shifting toward lower frequencies $\left(\sim 8 \mathrm{~cm}^{-1}\right)$ and a peak broadening are obtained on going from highly diluted MS in $\mathrm{CCl}_{4}$ toward pure liquid MS. The signals in the 2800-3500 $\mathrm{cm}^{-1}$ show the same characteristics of the $\mathrm{C}=0$ one: slight red-shifting $\left(\sim 7 \mathrm{~cm}^{-1}\right)$ and signal broadening.

The effect of temperature on intramolecular hydrogen bonding is reported in Figure 5 using the variation of the $\mathrm{C}=0$
ATR-FTIR peak. The maxima of this peak do not change with increasing temperature and only a weakening of peak intensity is obtained. Therefore, intramolecular hydrogen bonding remains in liquid MS up to high temperatures, as we may expect considering the gas phase calculations reported in the previous paragraph.

Therefore, these ATR-FTIR results points to i. Strong intermolecular hydrogen bonding between the hydroxyl hydrogen and carbonyl oxygen both in gas phase (obtained through DFT calculations), diluted in inert solvents such as $\mathrm{CCl}_{4}$ or in pure liquid MS with a weak effect of temperature and ii. Absence of remarkable intermolecular hydrogen bonding and thus no effect on the intramolecular interaction.

Finally, a DRS study in the microwave frequency region was performed on pure liquid MS as a function of temperature. The frequency dependence of the real, $\varepsilon^{\prime}$, and imaginary, $\varepsilon^{\prime \prime}$, parts of the complex dielectric function, $\varepsilon^{*}$, for pure MS is reported in Figure 6, in the 278.15-358.15 $\mathrm{K}$ temperature range. The analysis of experimental dielectric results was done according to a relaxation time distribution involving two Debye processes (eq. $1, k=2$ ):

$\varepsilon^{*}(v)=\sum_{i=1}^{k} \frac{\Delta \varepsilon_{i}}{1+i 2 \pi v \tau_{i}}+\varepsilon_{\infty}$

where $i$ is the imaginary unit, $v$ stands for the frequency, $\varepsilon_{\infty}$ is the dielectric constant in the high frequency limit, $\Delta \varepsilon_{i}$ the relaxation amplitude and $\tau_{i}$ the relaxation time. Fitting results are reported in Table 2 and Figure 7. The dielectric relaxation spectrum of pure MS shows a complex temperature variation, mainly for the real component. Nevertheless, a well defined peak in the imaginary component is obtained, that shifts toward higher frequencies (faster relaxation) as temperature rises. The two Debye terms required to describe pure MS dielectric relaxation behaviour shows also a complex temperature trend. The first Debye term, relaxation process 1 , is the most important one for almost the full temperature range, only for $T>328.15 \mathrm{~K}$ the second Debye term, relaxation process 2 , develops a relevant role. Process 1 appears at lower frequencies than process 2 , and thus, process 1 has to be assigned to a slower relaxation process. The intensity of process 1 decreases with increasing temperature, whereas for process 2 the inverse behavior is obtained, Figure 7. Moreover, relaxation time of process 1 also decreases with increasing temperature whereas for process 2 it evolves through a minimum. The data reported in Figure 7 for relaxation time of process 1 show that two regimes may be inferred for this 

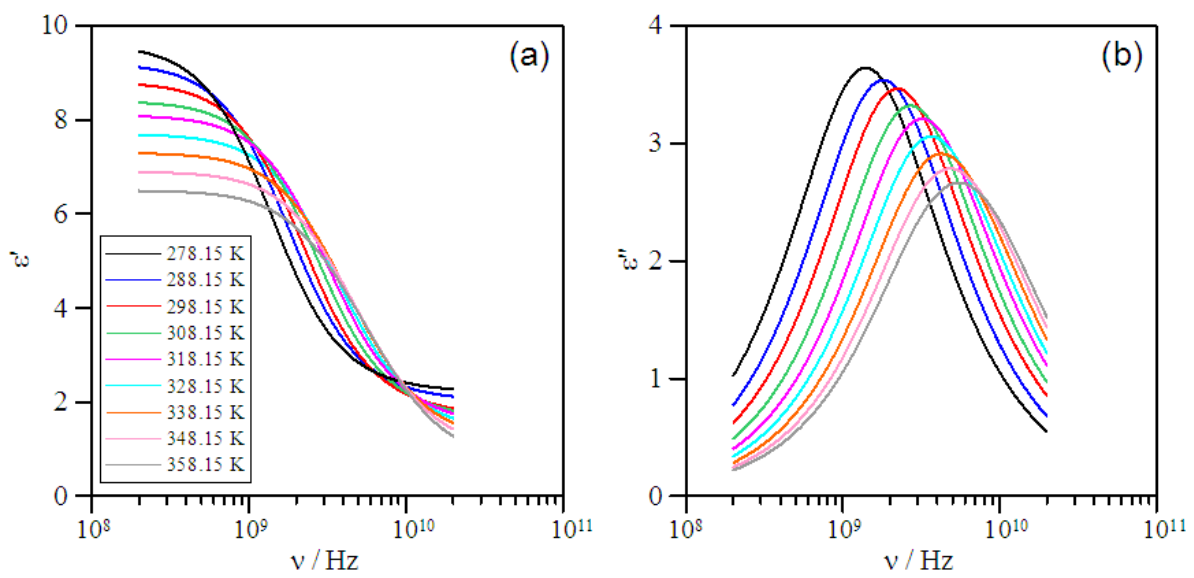

Figure 6. Real, $\varepsilon^{\prime}$, and imaginary, $\varepsilon^{\prime \prime}$, parts of the complex dielectric function of MS as a function of temperature. Solid lines are obtained considering two Debye processes and parameters from Table 1, experimental points are not included for the sake of clarity.
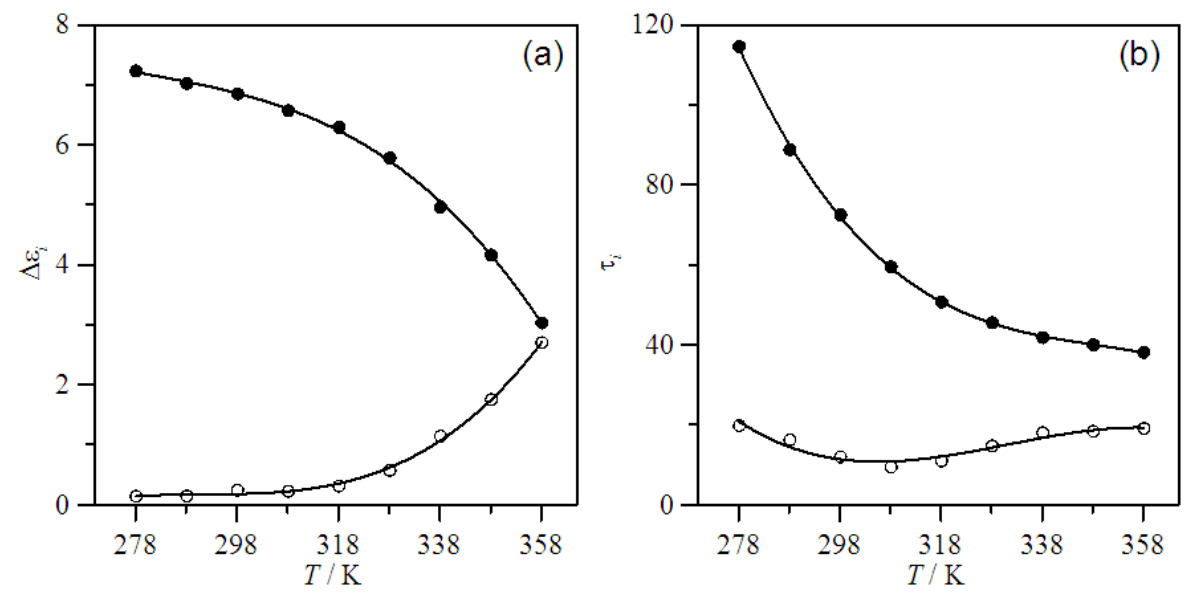

Figure 7. Relaxation amplitudes, $\Delta \varepsilon_{i}$ and relaxation times, $\tau_{i}$, obtained from the fit of experimental dielectric spectra according to two Debye processes, for MS as a function of temperature, $T$. Processes $(\bullet) 1$ and $(\circ) 2 ;(-)$ polynomial fittings for guiding purposes

process. In order to confirm these two separate zones for $\tau_{1}$, an analysis according to the Eyring equation was carried out:

$\ln \left(T \tau_{i}\right)=\left[\ln \left(\frac{h}{k}\right)-\frac{\Delta S^{\neq}}{R}\right]+\frac{\Delta H^{\neq}}{R T}$

where $\Delta H^{\ddagger}$ and $\Delta S^{\ddagger}$ are the molar enthalpy and entropy of activation respectively. Linear fits were done for $T \leq 318.15$ and for $T \geq 318.15 \mathrm{~K}$ from the data reported in Table 2 . Thus, for the slower relaxation time, $\tau_{1}, \Delta H^{\neq}=12.52 \mathrm{~kJ} \mathrm{~mol}^{-1}$ and $\Delta S^{\neq}=$$8.90 \mathrm{~J} \mathrm{~mol}^{-1} \mathrm{~K}^{-1}$ were obtained for $T \leq 318.15 \mathrm{~K}$ whereas $\Delta H^{\ddagger}=$ $2.82 \mathrm{~kJ} \mathrm{~mol}^{-1}$ and $\Delta S^{\neq}=-39.07 \mathrm{~J} \mathrm{~mol}^{-1} \mathrm{~K}^{-1}$ were obtained for $T \geq$ 318.15 K. Activation enthalpies are low in the whole temperature range, which is more remarkable for $T \geq 318.15 \mathrm{~K}$ and this relaxation process 1 evolves through a highly ordered transition state, as the negative values of activation entropies show. Therefore relaxation process 1 has to be assigned to the dwelling time of MS molecules, that is to say, the residence time of a MS molecule in a certain arrangement of neighbour MS molecules. Nevertheless, the low activation enthalpy together with the negative activation entropies favour the release of MS molecules, and thus, we may conclude that the interaction between neighbour molecules is not developed through intermolecular hydrogen bonding, that should lead to larger activation enthalpies. Hence intermolecular interactions should be developed mainly through dipolar interactions, and thus, allowing maintaining the strong intramolecular hydrogen bonding in agreement with results reported in previous sections. Nevertheless, the shape of MS molecules hinders the relaxation of MS molecules in clusters formed through dipolar interactions; this would justify the large values of $\tau_{1}$ that points to slow relaxation of MS molecules involved in clusters with neighbour molecules. As the temperature increases, the amplitude of this process decreases and the relaxation is faster, because considering that the interaction between neighbour molecules is developed mainly through dipolar interactions, these are remarkably weakened with increasing temperature, therefore the relaxation is faster, and the number of molecules involved in clusters is lower, therefore the amplitude decreases.

Table 2. Parameterization of experimental dielectric spectra according to two Debye processes for MS in the $200 \mathrm{MHz}-20 \mathrm{GHz}$ frequency range. Limiting high-frequency permittivity, $\varepsilon_{\infty}$, relaxation times, $\tau_{i}$ (for the $i$ Debye process), relaxation amplitudes, $\Delta \varepsilon_{i}$, and static dielectric constant, $\varepsilon_{\mathrm{s}}$.

\begin{tabular}{cccrrrrc}
\hline $\boldsymbol{T} / \mathbf{K}$ & $\boldsymbol{\Delta} \boldsymbol{\varepsilon}_{\boldsymbol{1}}$ & $\boldsymbol{\Delta} \boldsymbol{\varepsilon}_{\boldsymbol{2}}$ & $\tau_{\boldsymbol{1}} / \mathbf{p s}$ & $\tau_{\boldsymbol{2}} / \mathbf{p s}$ & $\boldsymbol{\varepsilon}_{\boldsymbol{s}}$ & $\boldsymbol{\varepsilon}_{\infty}$ & $\chi^{2}$ \\
\hline 278.15 & 7.23 & 0.14 & 114.57 & 19.74 & 2.22 & 9.6 & 0.723 \\
288.15 & 7.02 & 0.14 & 88.73 & 16.18 & 2.04 & 9.2 & 0.715 \\
298.15 & 6.85 & 0.24 & 72.46 & 11.95 & 1.71 & 8.8 & 0.628 \\
308.15 & 6.57 & 0.22 & 59.47 & 9.37 & 1.61 & 8.4 & 0.620 \\
318.15 & 6.29 & 0.31 & 50.64 & 10.92 & 1.50 & 8.1 & 0.739 \\
328.15 & 5.78 & 0.57 & 45.51 & 14.70 & 1.36 & 7.7 & 0.817 \\
338.15 & 4.96 & 1.14 & 41.80 & 17.97 & 1.20 & 7.3 & 0.952 \\
348.15 & 4.16 & 1.75 & 39.95 & 18.32 & 0.99 & 6.9 & 1.101 \\
358.15 & 3.03 & 2.70 & 38.10 & 19.03 & 0.77 & 6.5 & 1.263 \\
\hline
\end{tabular}


The assignment of relaxation process 2 is not simple, moreover considering its complex temperature behaviour. Nevertheless, we may consider that once a MS molecule is released from a cluster formed with neighbour molecules, it has to rotate to find a new favourable configuration, and thus, this fast relaxation would lead to process 2 . The increasing amplitude of this process 2 with increasing temperature may be explained considering that as the temperature rises the number of molecules involved in clusters is smaller and thus the rotation process is more important in comparison with the dwelling process attributed to process 1 in previous paragraph. $\tau_{2}$ evolves through a minimum with temperature $(\sim 308.15 \mathrm{~K})$. We may consider that up to $308-318 \mathrm{~K}$ the population of MS molecules involved in clusters, process 1, decreases remarkably whereas for larger temperatures its variation is not so remarkable, therefore up to $308-318 \mathrm{~K}$ rotation of MS molecules is more easy (faster relaxation) as temperature increases but for larger temperature the number of MS molecules not involved in clusters is very larger, which may hinder the rotation of MS molecules (slight shifting toward slower relaxation times). Therefore as main conclusions of DRS study we obtained $i$. Neighbour MS molecules do not interact through intermolecular hydrogen bonding and ii. Two relaxation processes may be inferred assigned to dwelling time of MS molecules in dipolar clusters and to free rotation of MS molecules.

\section{References}

[1]. Aparicio, S.; Alcalde, R.; Dávila, M. J.; García, B.; Leal, J. M. J. Phys. Chem. B 2007, 111, 4417-4431.

[2]. Aparicio, S.; Alcalde, R. Green Chem 2009, 11, 65-78.

[3]. Yamada, H. Bull. Chem. Soc. Jpn. 1959, 32, 1051-1056

[4]. Wojcik, M.J.; Paluszkiewicz, C. Can. J. Chem. 1983, 61, 1449-1452.

[5]. Berthelot, M.; Laurence, C.; Lucon, M.; Rossignol, C. J. Phys. Org. Chem. 1996, 9, 626-630.

[6]. Mitsuzuka, A.; Fujii, A.; Ebata, T.; Mikami, N. J. Phys. Chem. A 1998, 102, 9779-9784

[7]. Palomar, J.; De Paz, J. L. G.; Catalám, J. Chem. Phys. 1999, 246, 167-208.

[8]. Melandri, S.; Giuliano, B. M.; Maris, A.; Favero, L. B.; Ottaviani, P. Velino, B.; Caminati, W. J. Phys. Chem. A. 2007, 111, 9076-9079.

[9]. Massaro, R. D.; Dai, Y.; Blaistein-Barojas, E. J. Phys. Chem. A 2009, 113, 10385-10390.

[10]. Frisch, M. J.; Trucks, G. W.; Schlegel, H. B.; Scuseria, G. E.; Robb, M. A.; Cheeseman, J. R.; Montgomery, Jr., J. A.; Vreven, T.; Kudin, K.N.; Burant, J. C.; Millam, J. M.; Iyengar, S. S.; Tomasi, J.; Barone, V.; Mennucci, B.; Cossi, M.; Scalmani, G.; Rega, N.; Petersson, G. A.; Nakatsuji, H.; Hada, M.; Ehara, M.; Toyota, K.; Fukuda, R.; Hasegawa, J.; Ishida, M.; Nakajima, T.; Honda, Y.; Kitao, O.; Nakai, H.; Klene, M.; Li, X.; Knox, J.E.; Hratchian, H. P.; Cross, J.B.; Adamo, C.; Jaramillo, J.; Gomperts, R.; Stratmann, R. E.; Yazyev, O.; Austin, A. J.; Cammi, R.; Pomelli, C.; Ochterski, J. W.; Ayala, P. Y.; Morokuma, K.; Voth, G. A. Salvador, P.; Dannenberg, J.J.; Zakrzewski, V. G.; Dapprich, S.; Daniels, A. D.; Strain, M. C.; Farkas, O.; Malick, D. K.;Rabuck,A.D.; Raghavachari, K.; Foresman, J. B.; Ortiz, J. V.; Cui, Q.; Baboul, A. G.; Clifford, S.; Cioslowski, J.; Stefanov, B. B.; Liu, G.; Liashenko, A.; Piskorz, P.; Komaromi, I.; Martin, R. L.; Fox, D. J.; Keith, T.; Al-Laham, M. A.; Peng, C. Y.; Nanayakkara, A.; Challacombe, M.; Gill, P. M. W.; Johnson, B.; Chen, W.; Wong, M. W.; Gonzalez, C.; Pople, J. A. Gaussian 03 (Revision C.02), Gaussian, Inc., Wallingford CT, 2004.

[11]. Becke, A.D. Phys. Rev. A 1988, 38, 3098-3100.

[12]. Lee, C.; Yang, W.; Parr, R. G. Phys. Rev. B 1988, 37, 785-789.

[13]. Becke, A. D. J. Chem. Phys. 1993, 98, 5648-5652.

[14]. Bader, R.F.W. Atoms in Molecules: a Quantum Theory, Oxford University Press, Oxford, 1990.

[15]. Biegler-König, F.; Schönbohm, J.; Bayles, D. J. Comput. Chem. 2001, 22, 545-559.

[16]. Ponder, J. W. Tinker: Software tool for molecular design. 4.2 ed, Washington University School of Medicine, 2004.

[17]. Jorgensen, W. L.; Maxwell, D. S.; Tirado-Rives, J. J. Am. Chem. Soc. 1996, $118,11225-11236$.

[18]. Aparicio, S.; Alcalde, R.; García, B.; Leal, J. M. Chem. Phys. Lett. 2007, $444,252-257$

[19]. Koch, U.; Popelier, P. L. A. J. Phys. Chem. 1995, 99, 9747-9754.

[20]. Popelier, P. L. A. J. Phys. Chem. A 1998, 102, 1873-1878.

[21]. Hibbs, D.E.; Overgaard, J.; Piltz, R.O. Org. Biomol. Chem. 2003, 1, 11911198.

[22]. Espinosa, E.; Lecomte, C.; Molins, E. Chem. Phys. Lett. 1999, 300, 745-
748.

[23]. Espinosa, E.; Molins, E.; Lecomte, C. Chem. Phys. Lett. 1998, 285, 170173.

[24]. Varghese, H. T.; Panicker, C. Y.; Mannekutla, J. R.; Inamdar, S. R. Spectrochim. Acta A 2007, 66, 959-963. 